\title{
Age-associated pro-inflammatory adaptations of the mouse thoracic aorta
}

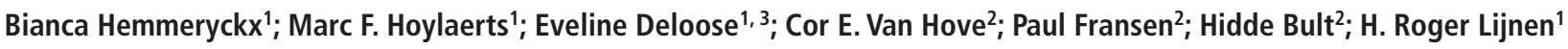 \\ ${ }^{1}$ Center for Molecular and Vascular Biology, KU Leuven, Leuven, Belgium; ${ }^{2}$ Division of Pharmacology, Universiteit Antwerpen, Wilrijk, Belgium; ${ }^{3}$ Present address: Translational \\ Research in Gastrointestinal Disorders, KU Leuven, Leuven, Belgium
}

\begin{abstract}
Summary
\section{Correspondence to:}

H. R. Lijnen

Center for Molecular and Vascular Biology

KU Leuven, Campus Gasthuisberg, CDG

Herestraat 49, Box 911, 3000 Leuven, Belgium

Tel.: +32 16345771 , Fax: +32 16345990

E-mail: roger.lijnen@med.kuleuven.be
\end{abstract}

Arterial ageing may be associated with a reduction in vasodilation due to increased reactive oxygen species (ROS) production, whereas endothelial cell activation induces procoagulant changes. However, little is known on the effect of ageing on expression of anticoagulant endothelial markers such as endothelial protein $C$ receptor (EPCR). To study age-associated alterations in smooth muscle cell (SMC) and endothelial cell (EC) structure and function, the aorta was isolated from 10-week- and 12- and 24-month-old C57BL/6J mice and analysed for its expression of genes involved in senescence, oxidative stress production, coagulation and matrix remodelling. In addition, vasorelaxation experiments were performed using 10-week- and 24-month-old thoracic aortic ring segments in organ chamber baths. The media thickness of the thoracic aorta progressively increased with age, as- sociated with hypertrophy of vascular SMCs. Basal nitric oxide production and sensitivity to acetylcholine-mediated vasodilation in thoracic aorta rings was reduced with age, whereas no significant differences in ROS production could be demonstrated. Gene expression of tissue factor, EPCR and von Willebrand factor was not affected by ageing of the aorta, whereas that of thrombomodulin was mildly reduced and that of xanthine dehydrogenase, NADPH oxidase 4, tumour necrosis factor- $\alpha$ and vascular cell adhesion molecule- 1 significantly enhanced. In conclusion, a reduction in endothelial cell-mediated vasodilation in aged thoracic aortas of C57BL/6J mice was accompanied by a shift towards a pro-inflammatory state of the endothelium.

\section{Keywords}

Vascular ageing, C57BL/6J mice, endothelial dysfunction, EPCR

Financial support:

The Center for Molecular and Vascular Biology is supported by the "Programmafinanciering Katholieke Universiteit Leuven" (PF/10/014). This study was supported by FWO project G.0678.10.

Received: January 10, 2013

Accepted after major revision: July 3, 2013

Prepublished online: August 8, 2013

doi:10.1160/TH13-01-0022

Thromb Haemost 2013; 110: 785-794

\section{Introduction}

The incidence of cardiovascular complications such as myocardial infarction is significantly increased with age, identifying age as a risk factor for arterial thrombosis (1). According to the updated triad of Virchow (2), this increased risk can be attributed to three factors: abnormalities in the vessel wall, in blood flow and in the coagulation, fibrinolytic and platelet systems.

Arterial ageing in humans is associated with increased collagen deposition, thickening of the intima-media of the human carotid artery, and reduced vascular elasticity. In addition, ageing of the aorta is accompanied by a reduction in endothelial cell-mediated vasodilation due to a decrease in nitric oxide (NO) bioavailability. The reduced NO levels result from an increased production of reactive oxygen species (ROS) such as superoxide anion $\left(\mathrm{O}_{2}{ }^{\circ}\right)$ with age. Simultaneous production of $\mathrm{NO}$ and $\mathrm{O}_{2}{ }^{--}$results in the generation of the cytotoxic product peroxynitrite anion $\left(\mathrm{ONOO}^{-}\right)$ (3). Enhanced production of $\mathrm{ONOO}^{-}$leads to a reduction in $\mathrm{NO}$ bioavailability and endothelial cell-mediated vasodilation and a decrease in anti-inflammatory and antithrombotic protection by $\mathrm{NO}$, causing more endothelium-platelet and endothelium-leukocyte interactions (4). ROS also upregulates endothelial nuclear factor kappaB (NF- $\mathrm{BB})$, aggravating prothrombotic tendencies as evidenced by upregulated expression of tissue factor (TF) in endothelial cells $(5,6)$. Other procoagulant changes are: 1$)$ a change in the balance of tissue plasminogen activator and its endogenous inhibitor plasminogen activator inhibitor-1 (7) and 2) von Willebrand factor (VWF) is released into the circulation by activated endothelial cells. VWF promotes platelet adhesion and aggregation and coagulation (8). However, age did not seem to influence the protein expression of the anticoagulant endothelial marker thrombomodulin (TM) in C57BL/6 mice in the carotid artery (9) or lungs (10). The endothelial protein $\mathrm{C}$ receptor (EPCR) is a receptor for activated protein $\mathrm{C}$ (APC), a serine protease, when activated by the thrombin-TM complex, prevents thrombosis through its direct anticoagulant activity: 1) APC can suppress 
thrombin generation by proteolytic cleavage of clotting cofactors FVa and FVIIIa, and 2) it can neutralise plasminogen activator inhibitor-1 leading to an increase in fibrinolytic activity (11). In addition, APC can prevent apoptosis, increase the endothelial barrier function and limit inflammation (12). Mutations in this gene have been associated with an increased risk for deep venous thrombosis (6936A/G polymorphism leading to increased soluble EPCR plasma levels, [13]) and to myocardial infarction (a 23 basepair insert [14]) in humans. In mice, APC displayed cardioprotective activities by decreasing the myocardial infarct size in isolated perfused hearts post-ischaemia via EPCR and protease-activated receptor 1-dependent signalling (15). The effect of age on the arterial expression of EPCR was investigated by us in hindlimb arterial blood vessels of C57BL/6J mice in a previous study (16). The expression of EPCR was, however not influenced by age (16), which was confirmed in another study showing that plasma active protein $\mathrm{Clev-}$ els were unchanged by age (10). As it has been suggested that the endothelial transcriptome of arteries at different anatomical locations can be different (17), we analysed the effect of age on the expression of EPCR in the thoracic aorta.

Therefore, we isolated the thoracic aorta from 10-week- and 12and 24-month-old C57BL/6J mice and analysed age-associated changes in smooth muscle cell and endothelial cell function. In addition, the aortic expression of genes involved in senescence, oxidative stress production, coagulation and matrix remodelling was monitored as a function of ageing.

\section{Materials and methods}

\section{Animal model}

Male C57Bl/6J mice at 10 weeks, 12 and 24 months of age were purchased from Janvier (Le Genest Saint Isle, France). All animals were kept in micro-isolation cages in a temperature- $\left(20-22^{\circ} \mathrm{C}\right)$ and light-controlled (12-hour night/day cycle) environment and ad libitum exposed to drinking water and standard chow (KM-04-k12, Muracon; Carfil, Oud-Turnhout, Belgium; 13 \% kcal as fat, caloric value $10.9 \mathrm{~kJ} / \mathrm{g}$ ). Mice were euthanised by intraperitoneal injection of $60 \mathrm{mg} / \mathrm{kg}$ sodium pentobarbital (Nembutal, Abbott Laboratories, North Chicago, IL, USA), and blood was retrieved from the retro-orbital sinus on $3.8 \%$ sodium citrate. Plasma was obtained by centrifuging the blood twice at 10,000 rpm for 10 minutes (min), and was stored at $-80^{\circ} \mathrm{C}$. Anesthetised mice were perfused with saline for $10 \mathrm{~min}$ to remove blood from the organs, and the thoracic aorta was isolated for immunohistological analysis. For RNA extraction and protein isolation purposes a larger aorta segment that encompassed the abdominal aorta and thoracic aorta was used. Aortic tissues were either immediately snap frozen in liquid nitrogen and stored at $-80^{\circ} \mathrm{C}$ or processed for the preparation of protein extracts. All animal procedures were approved by the Ethical Committee of the KU Leuven, and performed in accordance with the NIH Guide for the Care and Use of Laboratory Animals (1996).

\section{Isometric tension measurements}

Thoracic aorta rings were isolated from 10-week- and 24-monthold C57BL/6J mice $(\mathrm{n}=6)$ and two adjacent rings (width $2 \mathrm{~mm}$ ) from the central thoracic aorta were mounted in organ baths for isometric tension measurements, as described previously (18). One ring was continuously treated with $300 \mu \mathrm{M} \mathrm{N}^{\Omega}$-nitro-L-arginine (LNA) and $300 \mu \mathrm{M} \mathrm{N} \mathrm{N}^{\Omega}$-nitro-L-arginine methyl ester (LNAME), the other with buffer. Both rings first received cumulative phenylephrine (PE, $\left.10^{-9}-10^{-5} \mathrm{M}\right)$ concentrations. After three washes, both rings were constricted with $1 \mu \mathrm{M}$ PE followed by a cumulative concentration-response curve of diethylamine NONOate (DEANO) in the ring treated with LNA/LNAME, or of acetylcholine (ACh) in the other segment. The difference in maximum responses between adjacent rings without and with LNA/ LNAME was determined as an index of basal NO release (19). Responses to vasodilators were expressed as percentages of the initial contractions. Maximal responses and pD2 (negative logarithm of concentration causing $50 \%$ of maximal response) were determined for each segment using four parameter logistic regression.

\section{Histology and immunohistochemistry}

Thoracic aorta tissues were fixed in Shandon Zinc Formal-Fixx (Anatomical Pathology International, Chester, UK) for 20 hours (h), embedded in paraffin and $8 \mu \mathrm{m}$ cross-sections were prepared. Staining with hematoxylin and eosin (HE) or with Sirius Red was performed as described (20). Aortic sections were also stained with a rat anti-mouse CD31 antibody $(1 \mu \mathrm{g} / \mathrm{ml}, 557355 \mathrm{Mec} 13.3$, BD Biosciences, Erembodegem, Belgium), a rabbit anti-human VWF antibody (6.2 $\mu \mathrm{g} / \mathrm{ml}$; A0082 DAKO, Heverlee, Belgium), a goat anti-mouse (GAM) 409 TM antibody $(1 \mu \mathrm{g} / \mathrm{ml})$, a GAM EPCR antibody $(2.4 \mu \mathrm{g} / \mathrm{ml})$, a rabbit anti-mouse (RAM) nitrotyrosine (NT) antibody ( $4 \mu \mathrm{g} / \mathrm{ml}$; 06-284 Millipore, Brussels, Belgium), a GAM vascular cell adhesion molecule-1 (VCAM-1) antibody (2 $\mu \mathrm{g} / \mathrm{ml}$; AF643, R\&D Systems, Abingdon, UK), a GAM intercellular adhesion molecule-1 (ICAM-1) antibody $(0.8 \mu \mathrm{g} / \mathrm{ml}$; AF796, R\&D Systems), a rabbit anti-human myeloperoxidase (MPO) antibody (32 $\mu \mathrm{g} / \mathrm{ml}, A 0398, D A K O)$, and a RAM F4/80 $(10 \mu \mathrm{g} / \mathrm{ml}$, MCA497G, Serotec) diluted in Tris-NaCl-Blocking buffer (TNB) (TSA BT kit, NEL700001, Perkin Elmer Life Sciences), supplemented with (TM, EPCR) or without (CD31, VWF, VCAM-1, ICAM-1, NT, MPO, F4/80) pre-immune mouse serum (PIM). Negative controls included a section incubated with secondary antibodies alone or a section pre-incubated with $10 \mathrm{mM} 3-\mathrm{NT}$ for $10 \mathrm{~min}$. A positive control for the NT staining was obtained by chemically nitrating tyrosine residues with a $1 \mathrm{mM}$ sodium nitrite solution in $100 \mathrm{mM}$ sodium acetate $\mathrm{pH} 5.0$, supplemented with 1 $\mathrm{mM}$ hydrogen peroxide for $20 \mathrm{~min}$ at room temperature (RT). All secondary antibodies and pre-immune serums were purchased from DAKO. Detailed protocols for the CD31, VWF, TM and EPCR stainings were described previously (18). To expose NT, F4/80 and MPO antigens, sections were treated with antigen retrieval solution (DAKO) for $20 \mathrm{~min}$ at $95^{\circ} \mathrm{C}$ and were then allowed to cool. Subsequently, endogenous peroxidase activity was blocked 
by incubating sections (VCAM-1, ICAM-1, NT, F4/80) for 20 min at RT with methanol supplemented with $0.3 \% \mathrm{H}_{2} \mathrm{O}_{2}$. Aspecific binding to the biotin-labelled rabbit anti-goat (RAG) $(5.3 \mu \mathrm{g} / \mathrm{ml}$, VCAM-1, ICAM-1, DAKO), the biotin-coupled goat anti-rabbit (GAR) antibody (3.7 $\mu \mathrm{g} / \mathrm{ml}, \mathrm{NT}, \mathrm{DAKO})$, the biotin-labelled rabbit anti-rat antibody $(2.8 \mu \mathrm{g} / \mathrm{ml}, \mathrm{F} 4 / 80$, DAKO), and the horse radish peroxidase (HRP)-coupled swine anti-rabbit antibody $(26 \mu \mathrm{g} / \mathrm{ml}$, MPO, DAKO) was blocked by incubating sections, before application of the primary antibodies, with $20 \%$ pre-immune rabbit (VCAM-1, ICAM-1, F4/80), goat (NT), and swine (MPO) serum (DAKO) for $45 \mathrm{~min}$. VCAM-1, ICAM-1, and NT signals were visualised after incubation with HRP-coupled streptavidin (1/100; 30 min in TNB, RT, NEL75000EA, Perkin Elmer Life Sciences), and with 3, 3'-diaminobenzidine (DAB) (Sigma-Aldrich, Bornem, Belgium) at RT. An amplification step using biotinyl tyramide (1/50, NEL75000EA, Perkin Elmer Life Sciences) was added before DAB incubation for the F4/80 staining. MPO signals were immediately detected with DAB (Sigma-Aldrich).

\section{Western blotting}

Aorta tissues were isolated and immediately dipped in $130 \mu$ ice cold protein extraction buffer $\left(10 \mathrm{mM} \mathrm{Na}_{2} \mathrm{PO}_{4}, 0.15 \mathrm{M} \mathrm{NaCl}, 1 \%\right.$ Triton-X-100, $0.1 \%$ sodium dodecyl sulphate, $0.5 \% \mathrm{Na}$ deoxycholate, $0.2 \% \mathrm{Na}$ azide) supplemented with $50 \mathrm{mM}$ EDTA and 1/100 Halt protease and phosphatase inhibitor cocktail (78442, Thermo Fisher Scientific, Erembodegem, Belgium). Tissues were homogenised using a Fast-Prep-24 ribolyser (MP Biomedicals, Brussels, Belgium), subsequently centrifuged at $4^{\circ} \mathrm{C}$ for $20 \mathrm{~min}$ at 13,200 rpm, after which the supernatant was collected, and the protein concentration was determined using a standard bicinchoninic acid assay (Thermo Fisher Scientific). A total of $50 \mu \mathrm{g}$ (p16INK4a) or $100 \mu \mathrm{g}$ aorta extract isolated from three 10-week-old, three 12(only 2 for Phospho (P)-Akt/Akt) and three 24-month-old C57BL/6J mice was loaded and resolved on a NUPAGE 4-12\% Tris-Bis gel (TM, MPO, Life Technologies, Gent, Belgium), a NUPAGE 10\% Tris-Bis gel (p16INK4a, Life Technologies) or a 10\% Tris-glycine gel (P-Akt/Akt, Life Technologies). Proteins were electrophoretically transferred onto nitrocellulose membranes using the iBlot ${ }^{\oplus}$ semi-dry blotting system (Life Technologies). To avoid aspecific background signal, membranes were blocked for $4-6 \mathrm{~h}$ in TBS $(0.9 \% \mathrm{NaCl}, 10 \mathrm{mM}$ Tris-HCl, $\mathrm{pH}$ 7.4) supplemented with $0.01 \%$ Tween-20 (TBST) and 5\% blotting grade non-fat milk (BioRad Laboratories N.V., Nazareth Eke, Belgium). Membranes destined for incubation with TM and MPO antibodies were cut in two using the $70 \mathrm{kDa}$ band as a reference (lower part will be used to detect the housekeeping protein $\beta$-actin). Membranes were then incorporated overnight $(\mathrm{O} / \mathrm{N})$ at $4^{\circ} \mathrm{C}$ with the following antibodies diluted in TBST (P-Akt) or TBST supplemented with 5\% non-fat milk: 1/200 mouse monoclonal anti-p16INK4a antibody (sc-1661, Santa Cruz Biotechnologies Inc., Heidelberg, Germany), 1/400 GAM TM antibody (AF3894, R\&D Systems), 1/200 GAM MPO antibody (AF3667, R\&D Systems), and 1/1,000 rabbit polyclonal P-Akt (Ser473) antibody (9271, Cell Signalling, Boston, MA, USA). The membranes were washed and further incubated with
HRP-conjugated GAR antibodies (1/2,000, DAKO) diluted in TBST (P-Akt) for $2 \mathrm{~h}$ at RT, or with HRP-coupled GAM antibodies $(1 / 2,000$, p16INK4a, DAKO) and with HRP-coupled RAG antibodies (1/2,000, TM, MPO, DAKO) for $45 \mathrm{~min}$ at RT. Chemiluminescent detection was performed using regular (p16INK4a, TM, Akt) or strong (MPO, P-Akt) enhanced chemiluminescence (ECL) detection reagents (Thermo Fisher Scientific) and visualised using the ImageLab ${ }^{\text {Tw }}$ software Version 4.0 and the ChemiDoc ${ }^{\text {Tw }} \mathrm{XRS}^{+}$imager (Bio-Rad Laboratories N.V.). Membranes that have been incubated with antibodies directed against p16INK4a, and P-Akt were stripped using a mild stripping solution (Chemicon International Inc., Temecula, CA, USA). Membranes were either blocked O/N (p16INK4a, MPO, TM) or for $5 \mathrm{~h}$ (P-Akt) with TBST supplemented with $5 \%$ non-fat dry milk and reprobed with anti$\beta$-actin antibodies (p16INK4a membrane) for $2 \mathrm{hr}$ at RT in TBST supplemented with 5\% non-fat dry milk (1/500, 13E5, Cell Signalling) or with a rabbit polyclonal Akt antibody $(1 / 1,000,9272$, Cell Signalling) $\mathrm{O} / \mathrm{N}$ at $40^{\circ} \mathrm{C}$ in TBST. To visualise protein bands, GARHRP antibodies $(1 / 2,000$, DAKO) in TBST (Akt) or TBST supplemented with $0.5 \%$ milk ( $\beta$-actin) were applied at RT for $45 \mathrm{~min}$ ( $\beta$-actin) or $2 \mathrm{~h}$ (Akt). Chemiluminescent detection was performed as described above. Regular ECL detection reagents were used to detect Akt protein levels, while a strong ECL solution was used to detect $\beta$-actin protein levels. Intensity of all proteins was determined by densitometric analysis using NIH Image J software (National Institutes of Health, Bethesda, MD, USA).

\section{Analyses}

Soluble VCAM-1 (sVCAM-1, MVCOO, R\&D Systems) and thiobarbituric acid reactive substances (TBAR5, KGE013, R\&D Systems) levels in plasma samples were analysed using a commercially available ELISA kit according to manufacturer's instructions.

\section{Microscopy analysis}

H\&E stainings were analysed with a Zeiss AxioPlan 2 Imaging microscope (Zeiss, Jena, Germany), and pictures were taken with Axiovision Rel. 4.6. (Zeiss) at x100 magnification. The media thickness was assessed using KS300 software (Zeiss) by measuring the length of the media at 10 different positions in six different sections per animal. Vascular smooth muscle cell (VSMC) density was determined on H\&E stainings under normal light. Per mouse 10 different sections were analysed by measuring the total area of the aortic section, the total area of the lumen and the SMC count within the elastic media. The VSMC density was calculated as the ratio of the SMC number to the media area (area aortic section area lumen). Measurements were pooled to obtain an average value per animal, and the data for all animals in one group were then averaged. CD31, TM, VWF, EPCR, VCAM-1, ICAM-1 and F4/80 stainings were analysed with a Zeiss Axiovert 200M Imaging microscope (Zeiss), and pictures were taken with Axiovision Rel. 4.8. (Zeiss) at x100 magnification. The F4/80-positive media area in six different thoracic aorta sections per animal was determined by segmentation (NIH Image J software). The results were then 
averaged per animal and subsequently per group. The intensities of the CD31, TM, VWF, EPCR, VCAM-1, and ICAM-1 stainings were quantitated by measuring DAB pixel intensity using the NIH Image J software (21). The samples of each staining were blinded.

Table 1: Age-associated changes in expression of genes involved in regulation of different cellular processes in aortic tissues of C57BI/6J mice.

\begin{tabular}{l|l|l|l}
\hline \multicolumn{1}{|l}{} & 10 weeks & 12 months & 24 months \\
\hline Senescence & & & \\
\hline p16INK4a & $0.61 \pm 0.067$ & $1.2 \pm 0.22^{*}$ & $2.2 \pm 0.37^{*} \dagger$ \\
\hline Oxidative stress & & & \\
\hline Catalase & $4,840 \pm 370$ & $3,970 \pm 460$ & $5,100 \pm 1,010$ \\
\hline SOD1 & $3,870 \pm 240$ & $3,120 \pm 290$ & $3,970 \pm 380$ \\
\hline Gpx1 & $5,160 \pm 280$ & $4,530 \pm 320$ & $4,790 \pm 580$ \\
\hline Xdh & $1,790 \pm 120$ & $2,170 \pm 250$ & $3,160 \pm 500^{*}$ \\
\hline Nox4 & $2,880 \pm 110$ & $3,470 \pm 260^{*}$ & $2,820 \pm 290$ \\
\hline Coagulation & & & \\
\hline EPCR & $249 \pm 26$ & $245 \pm 32$ & $292 \pm 40$ \\
\hline TM & $1,662 \pm 149$ & $1,974 \pm 174$ & $1,505 \pm 98 \dagger$ \\
\hline VWF & $1,039 \pm 116$ & $808 \pm 132$ & $938 \pm 61$ \\
\hline TF & $452 \pm 42$ & $705 \pm 103$ & $491 \pm 63$ \\
\hline Inflammation & & & \\
\hline eNOS & $276 \pm 11$ & $224 \pm 41$ & $308 \pm 58$ \\
\hline iNOS & $17 \pm 1.7$ & $15 \pm 2.6$ & $24 \pm 5.8$ \\
\hline TNF- $\alpha$ & $2.0 \pm 0.24$ & $2.6 \pm 0.41$ & $5.1 \pm 0.82^{*} \dagger$ \\
\hline NF-KB & $400 \pm 30$ & $340 \pm 20$ & $310 \pm 20$ \\
\hline TGF- $\beta_{1}$ & $514 \pm 18$ & $590 \pm 51$ & $633 \pm 45^{*}$ \\
\hline MPO & $0.93 \pm 0.31$ & $1.1 \pm 0.34$ & $2.1 \pm 0.55$ \\
\hline ECM remodelling & $555 \pm 14$ & $688 \pm 73$ & $645 \pm 73$ \\
\hline MMP-2 & $719 \pm 86$ & $558 \pm 53$ & $639 \pm 111$ \\
\hline TSP1 & $257 \pm 24$ & $284 \pm 28$ & $244 \pm 23$ \\
\hline TSP2 & & & \\
\hline mRNA & & & \\
\hline
\end{tabular}

mRNA expression levels of the target genes are normalised to the expression level of GAPDH and subsequently expressed as the copy number of target mRNA relative to $10^{5}$ copies of GAPDH. Data are means \pm SEM of 8-10 determinations. Abbreviations: superoxide dismutase 1 (SOD1); glutathione peroxidase 1 (Gpx1); xanthine dehydrogenase (Xdh); NADPH oxidase 4 (Nox4); endothelial and inducible nitric oxide synthase (eNOS and iNOS); endothelial protein $C$ receptor (EPCR); thrombomodulin (TM); von Willebrand Factor (VWF); tissue factor (TF); tumour necrosis factor- $\alpha$ (TNF- $\alpha)$; nuclear factor kappaB (NF-KB); transforming growth factor $\beta_{1}\left(\mathrm{TGF}-\beta_{1}\right)$; myeloperoxidase (MPO); matrix metalloproteinase 2 (MMP-2); thrombospondin-1 and -2 (TSP1, TSP2). * $p<0.05$ vs 10 weeks; $\dagger p<0.05$ vs 12 months.

\section{Quantitative real time-PCR (RT-PCR)}

p16INK4a (Mm00494449_m1), catalase (Mm00437992_m1), superoxide dismutase 1 (SOD1, Mm01700393_g1), glutathione peroxidase 1 (Gpx1, Mm00656767_g1), xanthine dehydrogenase (Xdh, Mm00442110_m1), NADPH oxidase 4 (Nox4, Mm00479246_m1), endothelial NO synthase (eNOS, Mm00435217_m1), inducible NOS (iNOS, Mm01309902_m1), TF (Mm00438853_m1), VWF (Mm00550376_m1), TM (Mm00437014_s1), EPCR (Mm00440992_m1), tumour necrosis factor- $\alpha$ (TNF- $\alpha$, Mm00443258_m1), NF- $\mathrm{kB}$ (Mm00476379_m1), transforming growth factor (TGF) $-\beta_{1}$ (Mm03024053_m1), MPO $(\mathrm{Mm01298424}$ _m1)), matrix metalloproteinase-2 (MMP-2, Mm00439498_m1), thrombospondin-1 (TSP1, Mm00449022_m1) and TSP2 (Mm01279240_m1) Taqman expression assays were used to determine mRNA levels in aortic tissue by quantitative RT-PCR, as described elsewhere (18). Data were obtained as cycle threshold $(\mathrm{Ct})$ values of two replicates and expressed as copy number of target mRNA relative to $10^{5}$ copies of the housekeeping gene glyceraldehyde 3-phosphate dehydrogenase (GAPDH).

\section{Statistical analysis}

GraphPad Prism 4.03 software was used to determine statistical differences (GraphPad, La Jolla, CA). Data are shown as means \pm SEM. The non-parametric Mann-Whitney U-test was used to analyse differences between groups. Normally distributed concentration response curves in the vasodilation tests were analysed as a function of age with a two-way ANOVA, and other data of the isometric tension measurements with one-way ANOVA. Statistical significance was set at $\mathrm{p}<0.05$.

\section{Results}

\section{Morphological characteristics of ageing thoracic aorta}

With ageing, body weight of the C57Bl/6J mice increased from 24 $\pm 0.18 \mathrm{~g}$ at 10 weeks to $30 \pm 0.26 \mathrm{~g}$ at 12 months and $30 \pm 0.57 \mathrm{~g}$ at 24 months ( $n=20 ; p<0.0001$ at 10 weeks vs 12 - and 24-month-old mice). Enhanced gene expression of the senescence marker p16INK4a was confirmed in aorta extracts of 12 months (2.0-fold) and 24 months (3.6-fold) as compared to 10-week-old mice ( Table 1). This pattern in p16INK4a expression with age was confirmed by western blot ( Figure 1). The media thickness of the thoracic aorta progressively increased with age ( Figure 2A, B). The VSMC density, in contrast, progressively decreased with age ( $\triangleright$ Figure 2D), while the total VSMC number significantly decreased between the age of 10 weeks and 24 months ( Figure 2A, C). 

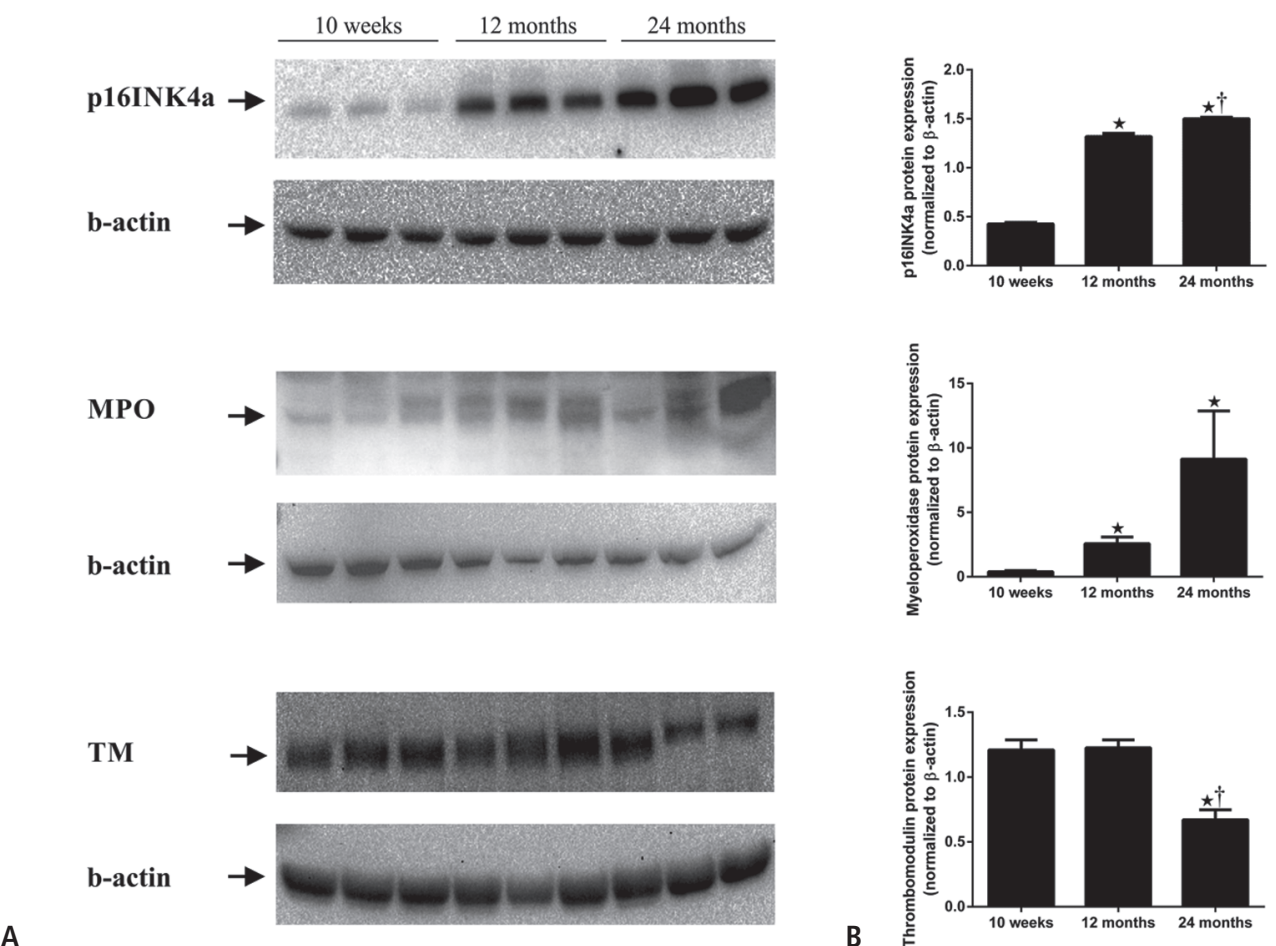

A

\section{B}

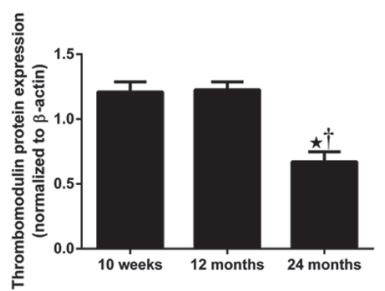

Figure 1: Influence of age on the protein expression of p16INK4a, myeloperoxidase and thrombomodulin in $\mathrm{C} 57 \mathrm{BL} / 6 \mathrm{~J}$ aorta extracts. A) 50 (p16INK4a) or $100 \mu \mathrm{g}$ protein extract of 10-week-, 12- and 24-month-old C57BL/6J mice ( $\mathrm{n}=3$ each) were analysed by western blotting for the expression of p16INK4a (sc-1661, 1/200), myeloperoxidase (MPO, 1/200,

\section{Smooth muscle and endothelial cell function of ageing thoracic aorta}

The effect of age on SMC and endothelial cell function was tested in thoracic aorta segments isolated from 10-week- and 24-monthold C57BL/6J mice. SMC contraction was studied in the presence of the NOS inhibitors LNA/LNAME. Maximal contractility was affected by age ( $\$$ Figure $3 \mathrm{~A}$, red curves), as aortic rings obtained from 24-month-old mice developed a higher maximum force in response to $\mathrm{PE}$ as compared to aortic segments from young animals. However, sensitivity to $P E$ was equal at both ages $\left(\mathrm{pD}_{2}\right.$ values at 10 weeks $6.88 \pm 0.12$; at 24 months $6.87 \pm 0.09, \mathrm{n}=6, \mathrm{p}>0.05)$.

In the presence of functional NOS ( Figure $3 \mathrm{~A}$, black curves), $\mathrm{PE}$ induced much less contraction compared to rings treated with NOS inhibitors. Interestingly, when NOS was active, PE evoked greater contractions in 24-month-old mice as compared to 10-week-old mice ( Figure 3A). The force difference at $10^{-5} \mathrm{M}$ $\mathrm{PE}$ between the ring with and the ring without NOS inhibitors provided an assessment of basal NO release ( Figure 3B). The effect of NOS inhibition was significantly greater in young C57BL/6 J mice.

Subsequently, ACh was given to activate NOS activity by stimulating endothelial muscarinic receptors. ACh caused almost complete relaxation at both ages; however, the vasodilator effect oc-
AF3667) and thrombomodulin (TM, 1/400, AF3894) with the corresponding $\beta$-actin $(1 / 500,13 E 5)$ protein expression for each blot. B) Densitometric analysis of panel $A$. Data are represented as means \pm SEM of nine measurements. ${ }^{*} p<0.05$ vs 10 weeks, $+p<0.05$ vs 12 months.

curred at lower ACh concentrations in rings collected at 10 weeks as compared to the older segments ( $\triangleright$ Figure 3D). This difference was also reflected in the sensitivity of Ach-induced relaxation $\left(\mathrm{pD}_{2}\right.$ values at 10 weeks $7.41 \pm 0.10$, at 24 months $7.04 \pm 0.06, n=6$, $\mathrm{p}<0.05)$.

To test whether reduced responsiveness of the SMC to NO contributed to the attenuated NOS-dependent vasodilator responses at old age, rings were constricted with PE in the presence of LNA/ LNAME and then exposed to DEANO. This donor of exogenous NO caused complete relaxation in all rings without differences for age ( $\triangleright$ Figure $3 \mathrm{C})$.

\section{Gene expression profile of ageing aorta}

Of the genes involved in regulation of oxidative stress that were investigated, a 1.8-fold upregulation of Xdh in 24-month-old aortas and a 1.2-fold upregulation of Nox4 in 12-month-old aortas was observed, as compared to 10 weeks. Expression of catalase, SOD1 and Gpx1 was not affected by age ( Table 1). Of the genes involved in endothelial biology/coagulation, expression of vascular TF, VWR, and EPCR was not affected by age, whereas expression of TM was 1.3 -fold downregulated at 24 as compared to 12 months of age. Of the genes involved in inflammation, significant upregulation at 24 months vs 10 weeks of age was observed for 


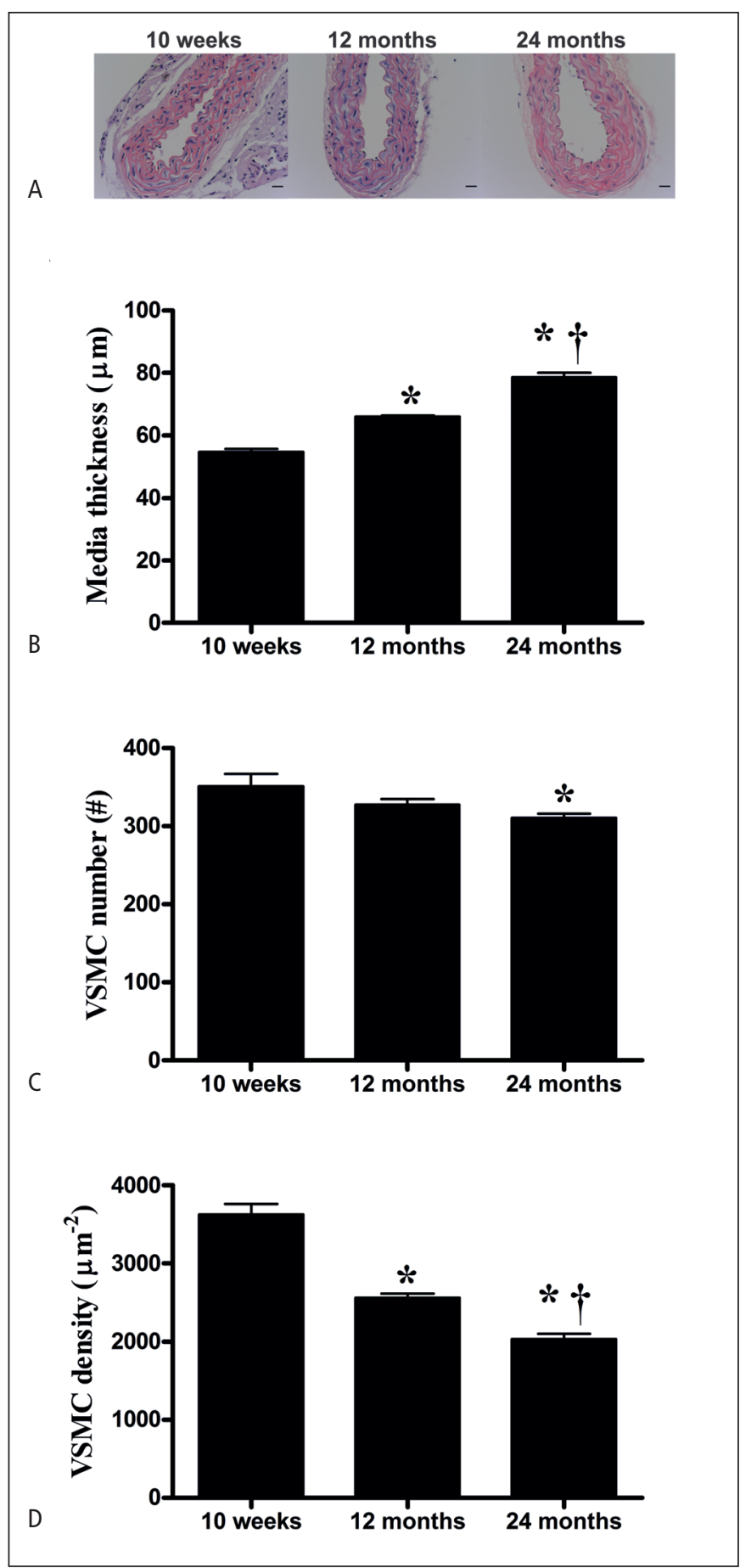

Figure 2: Influence of age on structure and morphology of the thoracic aorta. Paraffin-embedded aorta sections of 10-week-, 12- and 24-month-old C57BI/6J mice were stained with H\&E (A) and used for analysis of media thickness (B) and vascular smooth muscle cell (VSMC) number (C) and density (D). Data are means \pm SEM of 9-10 determinations. * $p<0.05$ vs 10 weeks, $\uparrow p<0.05$ vs 12 months. Magnification: 400x. Scale bar: $20 \mu \mathrm{m}$.
TNF- $\alpha$ (3.2-fold) and TGF- $\beta_{1}$ (1.2-fold), while mRNA levels of MPO tended to increase from the age of 10 weeks to 24 months (2.3-fold, $\mathrm{p}=0.08)$. This age-associated difference in MPO gene expression was confirmed by western blotting: 24-month-old aorta extracts showed a 23-fold higher MPO protein expression as compared to 10-week-old aorta extracts ( Figure 1). Expression of NF- $\mathrm{kB}$, eNOS and iNOS was not affected by age. Expression of the matrix metalloproteinase MMP-2 and the matricellular proteins TSP-1 and TSP-2 was not affected by age.

\section{Immunohistochemical analysis of ageing thoracic aorta}

Visualising the endothelial cell layer by CD31 staining did not reveal a different pattern with ageing ( Table 2). EPCR and VWF expression were not affected by ageing as well, compatible with the observed unaltered gene expression ( Table 1). MPO expression could not be detected in thoracic aorta sections of any age group and therefore could not be analysed (data not shown). The amount of infiltrating macrophages as assayed by a F4/80 staining was significantly increased in aorta sections of 12- and 24-month-old mice $\left(47,323 \pm 10,619 \mu \mathrm{m}^{2}\right.$ and $\left.25,317 \pm 6,168 \mu \mathrm{m}^{2}\right)$ in comparison to sections of 10 -week-old mice ( $\left.668 \pm 168 \mu \mathrm{m}^{2}, \mathrm{p}<0.01\right)$. ICAM-1 expression was significantly enhanced at 12 months of age, but not at 24 months. VCAM-1 expression was significantly enhanced at 24 months vs 10 weeks of age ( Table 2). The soluble form of VCAM-1 (sVCAM-1) is considered an early marker of endothelial activation. In concordance with the gene and protein expression pattern of VCAM-1 in aortic extracts, plasma sVCAM-1 levels progressively increased with age (10 weeks: $736 \pm 21 \mathrm{ng} / \mathrm{ml}$ vs 12 months: $872 \pm 36 \mathrm{ng} / \mathrm{ml}, \mathrm{p}=0.01$; and vs 24 months: $1,048 \pm 2.0 \mathrm{ng} /$ $\mathrm{ml}, \mathrm{p}=0.0004$ ). Paradoxically, expression of the anticoagulant TM was enhanced in the aorta of 24 months old mice ( Table 2), which is in apparent contrast with the observed downregulation of gene expression ( Table 1). However, when TM protein levels in aorta extracts were analysed by western blotting, a decrease in TM expression in 24-month-old mice was observed vs 10-week- and 12-month-old mice ( Figure 1), confirming the gene expression data.

\section{Oxidative stress levels with age}

Staining of thoracic aorta sections of each group with 3-nitrotyrosine antibodies did not show detectable 3-nitrotyrosine protein levels. In contrast, strong staining was observed in a thoracic aorta section, which was pre-incubated with nitrite and hydrogen peroxide before incubation with 3-nitrotyrosine antibodies (data not shown).

\section{Discussion}

Several procoagulant endothelial changes with age have already been described (5-6, 9-10); however, no effect of age was observed on the arterial expression of the anticoagulant endothelial marker 


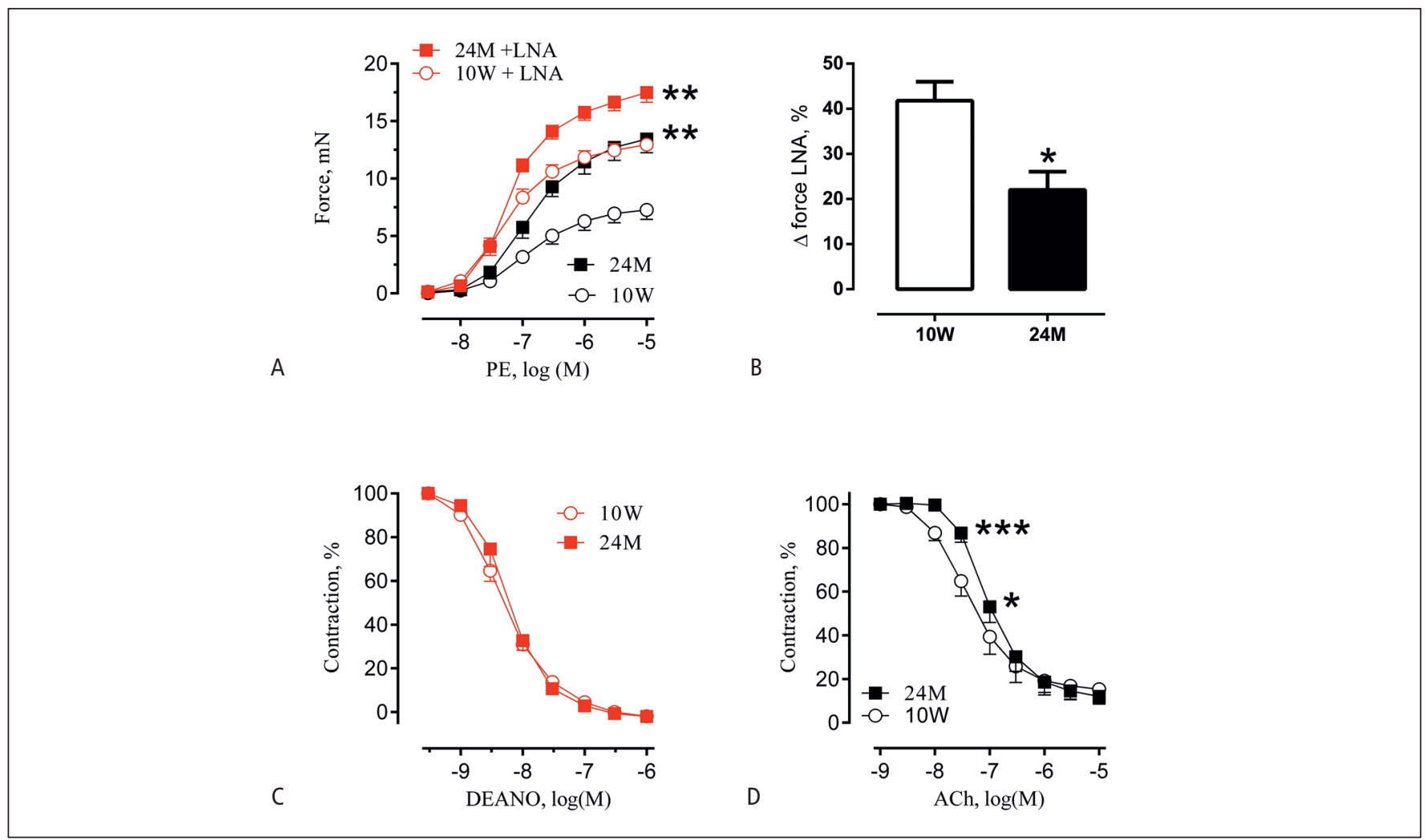

Figure 3: Effect of age on smooth muscle and endothelial cell function in aortic rings of $\mathrm{C} 57 \mathrm{BL} / 6 \mathrm{~J}$ mice. A) Contractions evoked by phenylephrine (PE) in the absence (black symbols, lower curves) and presence of the NOS inhibitors $\mathrm{N}^{\Omega}$-nitro-L-arginine $(\mathrm{LNA}, 300 \mu \mathrm{M})$ and $\mathrm{N}^{\Omega}$-nitro-L-arginine methyl ester (LNAME, $300 \mu \mathrm{M}$ ) (red, upper curves). Segments from older mice (24 months, 24M) developed more tension in both conditions vs young mice (10 weeks, $10 \mathrm{~W})$. B) The difference in force at $10^{-5}$

EPCR in hindlimb blood vessels of C57BL/6J mice (16). As it has been suggested that the endothelial gene expression profile of arteries at different anatomical locations can be different (17), we isolated the thoracic aorta from 10-week- and 12- and 24-monthold C57BL/6J mice and analysed age-associated changes in the procoagulant/anticoagulant phenotype of the arterial endothelium. To address the stage of vascular ageing, smooth muscle and endothelial cell structure and function were evaluated. In addition, the aortic expression of genes involved in senescence, oxidative stress production, and matrix remodelling was monitored as a function of ageing.

Ageing of the thoracic aorta was accompanied by hypertrophy and increased contractility of the VSMCs, as indicated by an increase in media thickness, a decrease in VSMC number and concomitantly VSMC density, and an increase in the PE-generated contractile force of VSMCs. In addition, 24-month-old mice showed an elevated mRNA and protein expression of p16INK4a, suggesting senescence of the VSMCs and/or endothelial cells. In man, a decrease in VSMC density due to hypertrophy and senescence of VSMCs has been observed (1). Ageing of the aorta in mice and humans is also accompanied with structural changes
M PE between the ring with and the ring without NOS inhibitors provided an assessment of basal NO release. The effect of NOS inhibition was greater in young C57BL/6 mice. C) Relaxation evoked by DEANO in rings constricted with $1 \mu \mathrm{M}$ PE in the presence of LNA/LNAME. D) Relaxation evoked by acetylcholine $(\mathrm{ACh})$ in rings constricted with $1 \mu \mathrm{M}$ PE was attenuated in old mice. Results are means $\pm S E M, n=6 .{ }^{*} p<0.05,{ }^{* *} p<0.01,{ }^{* * *} p<0.001$ vs 10 weeks.

such as aortic stiffening due to replacement of elastin fibers by collagen $(1,22)$. Increased collagen contents in the 24-month-old thoracic aorta sections were however not observed compared to 10-week-old sections (data not shown).

Ageing has been shown to be an independent risk factor for cardiovascular diseases (1). Endothelial function or NO-mediated vasorelaxation declines with advancing age due to a diminished NO bioavailability, resulting from 1) changed expression/activity of eNOS; 2) increased generation of ROS such as superoxide anion and/or 3) decreased anti-oxidant defense system $(3,4)$. Vasomotor measurements using thoracic aortic rings from 10 -week- and 24-month-old mice in this study indicated that basal NO production was compromised by age, and additionally 24 -month-old aortic segments showed a reduced sensitivity towards ACh-mediated vasodilation compared to 10 -week-old segments. Contradictory to the findings of van der Loo et al. (3), ageing was not associated with a blunted maximal ACh-mediated relaxation response. One possible explanation might be the difference in age and species between both studies. We used C57BL/6J mice at an age of 24 months, while van der Loo et al. used F1 (F344 x BN) rats of a much older age (32-35 months) (3). Since SMC responses to the 
Table 2: Age-associated changes in expression of endothelial cell markers in the aorta of $\mathrm{C} 57 \mathrm{BL} / 6 \mathrm{~J}$ mice.

\begin{tabular}{l|l|l|l}
\hline & 10 weeks & 12 months & 24 months \\
\hline VCAM-1 & $0.93 \pm 0.21$ & $1.8 \pm 0.37$ & $4.0 \pm 0.66^{*} \dagger$ \\
\hline ICAM-1 & $6.4 \pm 0.46$ & $7.7 \pm 0.14^{*}$ & $5.9 \pm 0.43 \dagger$ \\
\hline EPCR & $2.0 \pm 0.26$ & $2.2 \pm 0.19$ & $2.1 \pm 0.20$ \\
\hline CD31 & $1.7 \pm 0.71$ & $3.5 \pm 0.72$ & $2.3 \pm 0.66$ \\
\hline TM & $1.4 \pm 0.28$ & $2.2 \pm 0.54$ & $3.7 \pm 0.56^{*}$ \\
\hline VWF & $2.9 \pm 0.44$ & $3.6 \pm 0.37$ & $3.5 \pm 0.31$ \\
\hline
\end{tabular}

Data represent stained areas expressed as percentages of section areas, and are means \pm SEM of 8-10 determinations. Abbreviations are: vascular cell adhesion molecule-1 (VCAM-1); intracellular adhesion molecule-1 (ICAM-1); endothelial protein C receptor (EPCR); thrombomodulin (TM) and von Willebrand Factor (VWF). * $p<0.05$ vs 10 weeks; $\dagger p<0.05$ vs 12 months.

exogenous NO donor DEANO were not altered, these findings point to a decreased bioavailability of endogenous $\mathrm{NO}$ under basal and ACh-stimulated conditions in elderly mice. It is important to note that the level of PE-induced force has no impact on relaxations induced by ACh or exogenous $\mathrm{NO}$ in the mouse aorta (current DEANO results and [23]). Lower NO levels were not due to a change in the gene expression of eNOS or iNOS or activation/ phosphorylation status of Akt ( Table 1, data not shown), but may be due to an increase in ROS production as suggested by an elevated aortic expression of the pro-oxidant enzymes Nox4, Xdh and MPO. A limitation of this study is, however, that the age-induced increases in ROS concentrations in arterial vessels as suggested in the literature $(3,22,24)$, could not be confirmed. The level of ROS generation in thoracic aorta sections was evaluated by staining sections with a 3-NT antibody. Only detectable 3-nitrotyrosine signals were present after chemical modification of proteins on the sections using a mixture of hydrogen peroxide and nitrite. Van der Loo et al. (3), Fleenor et al. (22) and Kang et al. (24) were, however, able to show an age-induced increase in arterial vessels using more sensitive methods: immunogold labeling of 3-nitrotyrosine and visualisation via electron microscopy (3) as well as analysis of 3-nitrotyrosine levels in protein lysates $(22,24)$. Systemically, an age-associated increase in oxidative stress via measurement of plasma TBARS levels (indicative of oxygen radical-induced lipid peroxidation) could not be confirmed as well (data not shown).

Endothelial dysfunction can shift the endothelium towards a procoagulant phenotype. Endothelium of the aged (24 months) aorta did show a slightly lower gene expression level of the anticoagulant protein TM as compared to 12-month-old endothelium, whereas the TM protein expression analysed via western blot was lower in 24-month- vs 10-week- and 12-month-old aorta extracts. Specifically in thoracic aorta sections, TM expression, however, was increased in 24-month- vs 10-week-old mice. This discrepancy may lie in the fact that a larger aorta segment was used that included the abdominal aorta as well, for mRNA and protein (western blot) analysis. Stämpfli et al. (9) and Starr et al. (10) observed a similar TM gene and protein expression between young and aged C57BL/6 mice, respectively. Only when the mice were exposed to another stressor (endotoxaemia), TM levels were lower in aged vs young mice (10) and resulted in a procoagulant endothelial phenotype. This age-associated downregulation of TM expression and resulting decrease in plasma activated protein $\mathrm{C}$ levels explained the vulnerability of aged mice to acute endotoxaemia (10). One of the probable mediators responsible for the ageassociated suppression of TM during endotoxaemia is TNF- $\alpha$ (25-27). As we did observe a progressive increase in TNF- $\alpha$ mRNA levels with age in aorta extracts, it may explain the decrease of TM mRNA transcripts in 24- vs 12-month aortas. The expression of EPCR was previously investigated in the hindlimb arterial vessels of 10-week- and 24-month-old C57BL/6J mice (16), and in the present study the EPCR expression in the thoracic aorta was studied in these mice. Both studies showed no age-associated effect on EPCR expression in the different arterial vessels. These findings suggest no shift of the thoracic arterial endothelium to a procoagulant state with age.

Age-associated endothelial dysfunction can induce a shift towards a pro-inflammatory phenotype of the endothelium, which may lead to an increased gene expression of TNF- $\alpha$ and its targets (28-31). In this study, the endothelium shifted to a pro-inflammatory state, as evidenced by an increased expression of the inflammatory marker TNF- $\alpha$ with age, concomitantly with the expression of several targets of TNF- $\alpha$ : the inflammatory marker VCAM-1, and the pro-oxidant enzymes Nox 4 and Xdh. Additionally, protein levels of VCAM-1 in thoracic aorta sections were upregulated in 24-month- vs 10-week-old mice and resulted in a significant release of sVCAM-1 with age. In addition, NF- $\mathrm{kB}$ signalling can upregulate the endothelial expression of VCAM-1 (32). Therefore, the gene expression level of NF- $\mathrm{kB}$ was also analysed. Although NF- $\mathrm{BB}$ expression was not influenced by age (its activity was not measured), it is still possible that NF- $\mathrm{kB}$ is involved in the increased endothelial VCAM-1 expression with age. These findings, however, conflict with the absent age-associated pro-inflammatory changes in the carotid artery of C57BL/6 mice, as evaluated by Stämpfli et al. (9), which may be related to the different expression analysis (gene vs protein and/or different markers) and the difference in vascular anatomical location. Increased expression of VCAM-1, a cell adhesion molecule responsible for the adhesion and transmigration of neutrophils and monocytes into the vascular endothelium, would result in an enhanced presence of inflammatory cells in the aorta of aged mice. Neutrophils and monocytes secrete MPO, an enzyme responsible for the consumption of $\mathrm{NO}$ and production of ROS that oxidise tetrahydrobiopterin, a cofactor necessary for eNOS activity (33). In a study by Walker et al. (34), impaired endothelial function in healthy middle-aged and older adults was related to higher neutrophil, eosinophil and monocyte-based white blood cell count and was characterized by a reduced responsiveness to $\mathrm{NO}$ and increased MPO-associated reductions in tetrahydrobiopterin and NO bioavailability. In a previous study, we observed an increase in the number of circulating monocytes and neutrophils with age in 


\section{What is known about this topic?}

- The endothelial protein $C$ receptor is a receptor for activated protein C (APC), which prevents thrombosis through its direct anticoagulant activity, prevents apoptosis, increases the endothelial barrier function and limits inflammation.

- The plasma active protein $C$ levels are unchanged by age in C57BL/6 mice.

- The expression of endothelial protein $C$ receptor in the hindlimb arterial blood vessels of C57BL/6J mice is not influenced by age; however, in the descending thoracic aorta this has not been studied.

\section{What does this paper add?}

- Gene and protein expression of endothelial protein $C$ receptor, tissue factor and von Willebrand factor are not affected by ageing of the thoracic aorta in C57BL/6J mice.

- Vascular cell adhesion molecule 1 levels are enhanced in 24-month- vs 10-week-old thoracic aorta sections.

- Ageing of the thoracic aorta is accompanied by a shift towards a pro-inflammatory state of the endothelium.

C57BL/6 mice (16). Additionally, the protein expression of MPO as assessed by western blotting in aorta was increased with age, and the aortas of 12- and 24-month-old mice expressed significantly more $\mathrm{F} 4 / 80$, a macrophage marker, in comparison to 10 -week-old mice, thus supporting the published observations (34).

In conclusion, ageing of the thoracic aorta in C57BL/6J mice as indicated by an increase in the expression of the senescence marker p16INK4a was accompanied by hypertrophy of the SMCs, a small reduction in basal and ACh-mediated NO release, and a change towards a pro-inflammatory, but not to a procoagulant phenotype of the endothelium. As we have previously observed enhanced platelet numbers with age in C57BL/6J mice (16), the increased risk for arterial thrombosis with age may relate more to increased interactions between endothelial cell-attached leukocytes and platelets, than to a shift towards a procoagulant phenotype of the endothelium.

\section{Acknowledgements}

We acknowledge Annemie De Wolf, Christine Vranckx, and Sofie Helsen for excellent technical assistance. We thank Prof. Dr. Charles T. Esmon (Oklahoma Medical Research Foundation, OK, USA) for a kind gift of the TM and EPCR antibodies.

\section{Conflicts of interest}

None declared.

\section{References}

1. Wilkerson WR, Sane DC. Aging and thrombosis. Semin Thromb Hemost 2002; 28: 555-568.

2. Makin A, Silverman SH, Lip GYH. Peripheral vascular disease and Virchow's triad for thrombogenesis. Q J Med 2002; 95: 199-210.

3. van der Loo B, Labugger R, Skepper JN, et al. Enhanced peroxynitrite formation is associated with vascular aging. J Exp Med 2000; 192: 1731-1744.

4. Brandes RP, Fleming I, Russe R. Endothelial aging. Cardiovasc Res 2005; 66: 286-294.

5. Donato AJ, Eskurza I, Silver AE, et al. Direct evidence of endothelial oxidative stress with aging in humans in relation to impaired endothelium-dependent dilation and upregulation of nuclear factor-kappa B. Circulation Res 2007; 100: 1659-1666.

6. Kollander R, Solovey A, Milbauer LC, et al. Nuclear factor-kappa B (NFkappaB) component p50 in blood mononuclear cells regulates endothelial tissue factor expression in sickle transgenic mice: implications for the coagulopathy of sickle cell disease. Transl Res 2010; 155: 170-177.

7. Vaughan DE. PAI-1 and atherothrombosis. J Thromb Haemost 2005; 3: 1879-1883.

8. Reininger AJ. Function of von Willebrand factor in haemostasis and thrombosis. Haemophilia 2008; 14: 11-26.

9. Stämpfli SF, Akhmedov A, Gebhard C, et al. Aging induces endothelial dysfunction while sparing arterial thrombosis. Arterioscler Thromb Vasc Biol 2010; 30: 1960-1967.

10. Starr ME, Ueda J, Takahashi H, et al. Age-dependent vulnerability to endotoxemia is associated with reduction of anticoagulant factors activated protein $\mathrm{C}$ and thrombomodulin. Blood 2010; 115: 4886-4893.

11. Dahlback B, Villoutreix BO. The anticoagulant protein $C$ pathway. FEBS Lett 2005; 579: 3310-3316.

12. Esmon CT. Protein $C$ anticoagulant-anti-inflammatory effects. Semin Immunopathol 2012; 34: 127-132.

13. Chen XD, Tian L, Li M, et al. Relationship between endothelial cell protein C receptor gene 693A/G polymorphisms and deep venous thrombosis. Chin Med J (Engl) 2011; 124: 72-75.

14. Van de Water NS, French JK, McDowell J, et al. The endothelial protein C receptor (EPCR) 23 bp insert in patients with myocardial infarction. Thromb Haemost 2001; 85: 749-751.

15. Wang J, Yang L, Rezaie AR, et al. Activated protein C protects against myocardial ischaemic/reperfusion injury through AMP-activated protein kinase signalling. J Thromb Haemost 2011; 9: 1308-1317.

16. Hemmeryckx B, Emmerechts J, Bovill EG, et al. Effect of ageing on the murine venous circulation. Histochem Cell Biol 2012; 137: 537-546.

17. Zhang J, Burridge KA, Friedman MH. In vivo differences between endothelial transcriptional profiles of coronary and iliac arteries revealed by microarray analysis. Am J Physiol Heart Circ Physiol 2008; 295: H1556-H1661.

18. Hemmeryckx B, Van Hove CE, Fransen P, et al. Progression of the prothrombotic state in ageing Bmal1-deficient mice. Arterioscler Thromb Vasc Biol 2011; 31: 2552-2559.

19. Fransen P, Van Assche T, Guns PJ, et al. Endothelial function in aorta segments of apolipoprotein E-deficient mice before development of atherosclerotic lesions. Pflugers Arch 2008; 455: 811-818.

20. Luttun A, Lutgens E, Manderveld A, et al. Loss of matrix metalloproteinase- 9 or matrix metalloproteinase-12 protects apolipoprotein E-deficient mice against atherosclerotic media destruction but differentially affects plaque growth. Circulation 2004; 109: 1408-1414.

21. Konsti J, Lundin M, Joensuu H, et al. Development and evaluation of a virtual microscopy application for automated assessment of Ki-67 expression in breast cancer. BMC Clin Pathol 2011; 11: 3.

22. Fleenor BS, Seals DR, Zigler ML, et al. Superoxide-lowering therapy with TEMPOL reverses arterial dysfunction with aging in mice. Aging Cell 2012; 11 : 269-276.

23. Van Hove CE, Van der Donkt C, Herman AG, et al. Vasodilator efficacy of nitric oxide depends on mechanisms of intracelllar calcium mobilisation in mouse aortic smooth muscle cells. Br J Pharmacol 2009; 158: 920-930.

24. Kang LS, Reyes RA, Muller-Delp JM. Aging impairs flow-induced dilation in coronary arterioles: role of $\mathrm{NO}$ and $\mathrm{H}(2) \mathrm{O}(2)$. Am J Physiol Heart Circ Physiol 2009; 297: H1087-H1095. 
25. Lentz SR, Tsiang M, Sadler JE. Regulation of thrombomodulin by tumor necrosis factor - comparison of transcriptional and posttranscriptional mechanisms. Blood 1991; 77: 542-550.

26. Kume M, Hayashi T, Yuasa H, et al. Bacterial lipopolysaccharide decreases thrombomodulin expression in the sinusoidal endothelial cells of rats: a possible mechanism of intrasinusoidal microthrombus formation and liver dysfunction. J Hepatol 2003; 38: 9-17.

27. Conway EM, Rosenberg RD. Tumor necrosis factor suppresses transcription of the thrombomodulin gene in endothelial cells. Mol Cell Biol 1988; 8: 5588-5592.

28. Rajan S, Ye J, Bai S, et al. NF-kappaB, but not p38 MAP kinase, is required for TNF-alpha-induced expression of cell adhesion molecules in endothelial cells. Cell Biochem 2008; 105: 447-486.

29. Zhang H, Park Y, Wu J, et al. Role of TNF-alpha in vascular dysfunction. Clin Sci 2009; 116: 219-230.
30. Lesniewski LA, Durrant JR, Connell ML, et al. Salicylate treatment improves age-associated vascular endothelial dysfunction: potential role of nuclear factor kappaB and forkhead Box O phosphorylation. J Gerontol A Biol Sci Med Sci 2011; 66: 409-418.

31. Lesniewski LA, Durrant JR, Connell ML, et al. Aerobic exercise reverses arterial inflammation with aging in mice. Am J Physiol Heart Circ Physiol 2011; 301: H1025-H1032.

32. Stein S, Schäfer N, Breitenstein A, et al. SIRT1 reduces endothelial activation without affecting vascular function in ApoE-/- mice. Aging 2010; 2: 353-360.

33. Eiserich JP, Baldus S, Brennan ML, et al. Myeloperoxidase, a leukocyte-derived vascular NO oxidase. Science 2002; 296: 2391-2394.

34. Walker AE, Seibert SM, Donato AJ, et al. Vascular endothelial function is related to white blood cell count and myeloperoxidase among healthy middle-aged and older adults. Hypertension 2010; 55: 363-369. 